\title{
Managing the Intersection of Medical and Pharmacy Benefits
}

\author{
Robert C. McDonald, MD, MBA
}

\begin{abstract}
BACKGROUND: Because of the unique features of specialty pharmaceuticals and insurance plans, specialty pharmaceutical products can be paid through the pharmacy benefit or the medical benefit. While most pharmacists are very comfortable with the conventions of reimbursement in the pharmacy benefit, they are less familiar with the processes for payment in the medical benefit.
\end{abstract}

OBJECTIVE: To review the 2 parallel processes for payment of specialty pharmaceuticals, the pharmacy benefit and the medical benefit, and to compare and contrast these 2 processes.

SUMMARY: The medical benefit and pharmacy benefit processes for payment of specialty pharmaceuticals use different claim forms, product coding systems, pricing conventions, and contracts. Even though the services delivered can be identical, the financial aspects of paying for these services are quite different.

CONCLUSION: Pharmacists who are interested in entering the specialty pharmacy arena, either as a provider or manager of providers, need to understand the payment processes for specialty pharmaceuticals through both the pharmacy and medical benefits.

J Manag Care Pharm. 2008;14(4)(suppl S):S7-S11

Copyright@ 2008, Academy of Managed Care Pharmacy. All rights reserved.

Author

ROBERT C. MCDONALD, MD, MBA, is president and founder of Aledo Consulting, Inc., in Indianapolis, Indiana.

AUTHOR CORRESPONDENCE: Robert C. McDonald, MD, MBA, President and Founder, Aledo Consulting, Inc., 8395 Keystone Crossing, Suite 206, Indianapolis, IN 46240. Tel.: 317.413.2004;

Fax: 317.466.1917; E-mail: bmcdonald@aledoconsulting.com pecialty pharmaceuticals may be viewed from a number of different perspectives: the patients who are prescribed specialty pharmaceuticals, the organizations regulating specialty pharmaceuticals (the U.S. Food and Drug Administration [FDA]), the pharmaceutical companies developing specialty pharmaceuticals, and the payers who pay for specialty pharmaceuticals. This review will focus on describing the payer perspective in some detail. In particular, payers have specific and unique processes of payment for specialty pharmaceuticals through the pharmacy benefit and the medical benefit. While descriptions of pharmaceutical payment methods are relatively uncommon in pharmacy literature, examples of such publications exist. ${ }^{1}$ Literature comparing the expenditures of specific classes of specialty pharmaceuticals, such as multiple sclerosis medications, has also been previously reviewed. ${ }^{2}$ After describing and comparing these 2 pathways, we will discuss some of the novel tactics that payers are using to manage specialty pharmaceuticals.

Literature addressing the high-level differences between the claims reimbursement processes for specialty pharmaceutical services in the pharmacy benefit versus the medical benefit is less common. The operational standards of these 2 approaches have been documented by managed care organizations, but the processes for submitting a claim in the medical benefit is less thoroughly understood by pharmacy professionals. It is challenging enough to operate a successful pharmacy submitting claims solely through the pharmacy benefit. However, when pharmacists decide to distribute specialty pharmaceuticals, they need to appreciate that the option of submitting claims through the medical benefit is available and is a tactic being used by their competitors.

\section{Pharmacy Benefits/Medical Benefits}

During the last 80 years in the United States, health insurance has been an evolving and typically expanding business. ${ }^{3}$ Foundational programs in the insurance industry, such as Blue Cross Blue Shield, and employer prepaid programs, such as Kaiser Permanente, started by focusing on providing a predetermined number of hospital days to employees in return for a regular payroll deduction. ${ }^{4}$ Growing from these origins as prepaid hospitalization plans, prepayment of outpatient services, such as physicians' services, were added in the decades that followed. In the 1970s and 1980s, coverage for pharmacy services was added to health plan offerings as the scope of health plan offerings continued to grow

Pharmaceuticals are a relative newcomer to the covered benefits of health plans and occupy a rather unique position. A quick review of the benefits of 1 of the largest health insurance programs in the country, the Federal Employees Health Benefit Plan, identified the following benefit categories: ${ }^{5}$ 
- Services provided by a hospital or other facility and ambulance services

- Medical services and supplies provided by physicians and other health care professionals

- Surgical and anesthesia services provided by physicians and other health care professionals

- Emergency services/accidents

- Mental health and substance abuse benefits

- Prescription drug benefits

- Dental benefits

Hospitals began interacting with health plans and government health programs in the mid-20th century. As a result, their contracts today can include 50 or more years of history. Pharmacies, on the other hand, have a different type of contract than hospitals. For instance, patients have different copayment or coinsurance obligations at pharmacies compared with hospital care. While it may not be intuitive, the multiple parallel benefits based primarily on site of service or provider type leads to the situation such that a dose of hemophilia factor, for example, may be paid for in completely different ways. Payment differences can exist based on whether the treatment is used as part of an inpatient hospital stay, an outpatient hospital service, as part of a service received at a physician's office, or a service received from a pharmacy.

Traditionally, pharmacists have focused on managing successful pharmacies using the pharmacy benefit as the only source of reimbursement. Because pharmacies are able to contract with health plans as medical benefit providers (in a pharmacy capacity, in a supplier capacity, or in a durable medical equipment provider capacity), the opportunity exists for pharmacies to have simultaneous contracts in the pharmacy and the medical benefit. This tactic is usually not important in the retail pharmacy setting, but can be very significant for a specialty pharmacy. Specialty pharmacies have commonly pursued both payment processes as a business practice; with the area of specialty pharmacy growing at a rapid rate, the importance of this issue is increasing.

\section{Magnitude of the Specialty Pharmacy Issue for Health Plans}

How health plans manage pharmaceuticals and specialty pharmaceuticals, in particular, has been a rapidly changing issue. This issue has evolved as pharmaceuticals have become a large and rapidly growing portion of the total health care expenditures; specialty pharmaceuticals has become the most rapidly growing portion of pharmacy expenditures. ${ }^{5}$ One of the specific challenges in calculating the exact expenditure on specialty pharmaceuticals is that their expenditures are split between the pharmacy benefit and the medical benefit. When only the specialty pharmaceuticals that are adjudicated through the pharmacy benefit are accounted for, these products make up $9.2 \%$ of the pharmacy benefit drug expenditure. ${ }^{5}$ When total U.S. pharmaceutical sales data are used to calculate sales of specialty pharmaceuticals, these products comprise about 20\% of total pharmaceutical expenditures. ${ }^{6}$ This difference exists because half of the specialty pharmaceuticals in the United States are not paid for in the pharmacy benefit, but rather are reimbursed through the outpatient or inpatient medical benefit.

It is important to note that total health care expenditures in the United States for 2005 were approximately \$1.988 trillion dollars, with $32 \%$ of the costs attributable to hospital care, $21 \%$ to physician/clinical services, and $10 \%$ to sales of retail prescription drugs. ${ }^{6}$ Within the pharmaceutical category, the specialty pharmaceutical category is growing even more rapidly than the pharmaceutical category as a whole. When compared with 2005 expenses, the expenses for specialty pharmaceuticals in 2006 were up 20.9\%; this compares with a 5.9\% trend for nonspecialty pharmaceuticals. ${ }^{6}$ Specialty pharmaceuticals are getting attention as their own class of drugs because they comprise about $2 \%$ of total health care expenditures, and their costs are growing at 4 times the rate of non-specialty pharmaceuticals.

There are many ways to define specialty pharmaceuticals. Some use the term "biotechnology products," which typically refers to peptide products developed with recombinant technology. While that was a very appropriate definition of specialty pharmaceuticals in the 1990s, there are several non-peptide injectable products that fall into the category of specialty pharmaceuticals (e.g., treprostinil sodium), and there are now oral specialty pharmaceuticals for rare diseases (e.g., bosentan and imatinib). Still other specialty pharmaceuticals require special handling because of perishability (e.g., biologics, vaccines, etc.) or radioactivity (e.g., tositumomab and ibritumomab tiuxetan). Therefore, the criteria of what constitutes a specialty pharmaceutical is continuing to evolve and expand in scope; all estimates are that this trend will continue drawing added health plan focus and resources. ${ }^{5,6}$ The details of how the underlying benefit structures have evolved over the past few decades is critical to understand.

\section{Parallel (Not Overlapping) Benefits}

Health plans' obligations to their members are structured as benefit language. Pharmacists typically understand the language of the pharmacy benefit. Fewer pharmacists understand how the current state of most benefit language results in a situation of overlapping pharmacy and medical benefits for specialty pharmaceuticals. Each chapter of an insurance contract lists categories of health care services that are covered by the health plan, and how each of those categories will be paid. Each of these sections describes a specific benefit; each benefit is defined, for the most part, by the location of where the service is delivered (e.g., hospital inpatient) or the provider type (e.g., physician). Pharmacies have a place in this hierarchy in 2 ways. First and foremost, they are providers in the pharmacy benefit, usually defined as the retail or mail-order setting provision of pharmacy services involving a drug card. Second, the pharmacy is an acceptable provider type under the medical benefit. In a sense, the pharmacy as a provider under the medical benefit is similar to a durable medical 
equipment provider or supplier that ships medical equipment to patients, typically at their homes. Therefore, there are 2 possible benefits and 2 possible contracts that a pharmacy could choose to pursue. This really is not a situation of blurring of the benefits, but rather that there are 2 parallel benefits, either of which can make business sense for the pharmacy. To enter the specialty pharmacy space effectively, a pharmacist is going to have to understand both processes and both sides of this issue.

These parallel benefits require additional analysis. As seen in Table 1, there are 2 parallel processes for the 2 different benefits that require the pharmacy to have contracts with different entities. The pharmacy benefit contracts are with the pharmacy benefits managers (PBMs), while the medical benefit contracts are directly with the health plans. If we use the metaphor of this process as being bilingual, this is the step at which it becomes bilingual. PBMs and health plans have industry standard terms and processes, and these terms may not have the same definition in the 2 different worlds. The health plan may have different certification requirements or other requirements than a PBM and vice versa. For instance, a PBM may have 1 definition of a pharmacy, and the health plan may have a different definition. Also, the claims payment systems are different for the pharmacy and medical benefits; the pharmacy benefit uses the National Council for Prescription Drug Programs (NCPDP) claim standard, while the medical benefit uses the Centers for Medicare \& Medicaid Services (CMS) 1500 claim form, formerly known as a Health Care Finance Administration (HCFA) 1500.

Another difference is the coding language: National Drug Codes (NDC) is used on the pharmacy benefit and Healthcare Common Procedure Coding System (HCPCS) is used on the medical benefit. The example of the home blood glucose meter can clarify the magnitude of the difference in philosophy of these 2 systems. There are hundreds of varieties of home blood glucose meters with each brand and model having a unique NDC code and price in the pharmacy claims payment system. In the medical claim system, these meters are represented by just 1 code (E0607), and typically they are reimbursed a single price. Also, standard pricing files used in the 2 benefits can be different. In the pharmacy benefit, it is common for PBMs to receive weekly or monthly updates of national standard pricing files that populate the claims payment engine. Adjusting prices in the medical benefit is a much less dynamic process with prices only updated annually in many cases. There are many other differences between these claims payment processes, including locations for submission, time to payment, etc.

A final difference is in the process of being harmonized: the provider ID numbers are used by the 2 systems. The provider numbers have been different between the 2 systems for decades, with pharmacy systems using National Association of Board of Pharmacy (NABP) numbers, and the medical systems typically using either a proprietary system or Tax Identification Number (TIN). As part of the Health Insurance Portability

\section{TABLE Detailed Comparison of Elements of Process for Pharmacy Claim Payment in Pharmacy and Medical Benefits}

\begin{tabular}{l|c|c}
\hline Benefit Type & Pharmacy & Medical \\
\hline $\begin{array}{l}\text { Unique provider } \\
\text { contracts }\end{array}$ & Yes, with PBM & Yes, with health plan \\
\hline $\begin{array}{l}\text { Provider type } \\
\text { Unique claim type }\end{array}$ & Pharmacy & $\begin{array}{c}\text { Pharmacy, supplier, } \\
\text { home health care }\end{array}$ \\
\hline Coding language & NDC & CMS 1500 \\
\hline Pricing source & $\begin{array}{c}\text { MediSpan Redbook } \\
\text { FirstDataBank }\end{array}$ & \begin{tabular}{c} 
Medicare or contract \\
\hline Provider number
\end{tabular} \\
\hline
\end{tabular}

CMS = Centers for Medicare $\&$ Medicaid Services; $H C P C S=$ Healthcare Common Procedure Coding System; NABP = National Association of Boards of Pharmacy; NCPDP $=$ National Council for Prescription Drug Programs; NDC = National Drug Code; NPI=National Provider Identifier; $P B M=$ pharmacy benefits manager .

and Accountability Act (HIPAA), medical payers have had to migrate to the National Provider Identification (NPI) number, and pharmacy claims payment systems are currently finishing their migration to this system. Many pharmacies find it daunting enough to manage the activities of the pharmacy benefit world without extending into the medical benefit world with its very different processes.

\section{The Evolution of Specialty Pharmacy: For Better and For Worse}

With the proliferation of specialty pharmaceuticals in the market and the number of differences between the ways that they can be paid on the different benefits, inefficiencies in payment practices have crept into the system.

For example, recombinant erythropoietin can be administered in a myriad of ways: as part of a hospitalization, as part of a stay in the hospital outpatient chemotherapy infusion clinic, as part of a doctor office visit for chemotherapy infusion, in the setting of a dialysis facility, or self-administered by a patient who receives the product on a pharmacy benefit. The payment rules for each of these categories are governed by different contracts and different benefit-driven payment terms. Each category of provider typically has its own contract type; it is not uncommon for 1 provider entity to have multiple contracts with a single health plan, each with a unique identity, provider type, and terms. The advent of the NPI system should make it easier for health plans to identify all the contractual relationships with a single provider. In addition, there are different sets of rules that apply to the different types of providers. Providers can set up an environment of varied contracts because it gives them multiple claims systems upon which to submit claims and to determine payment. One of the 
most common combinations is for a provider to be a pharmacist at the same time that they are a medical supplier.

Health plans have created an interesting situation as they have tried to move services out of the hospital and into the home-care setting over the last 2 decades. In many cases, plans have created lower copayments to patients when services were provided in the outpatient rather than the inpatient setting. ${ }^{7}$ Then, to create an incentive for moving the hospital outpatient services to the homecare setting, some plans have created incentives for patients to receive care in their homes. A home-care service may include the shipping of a specialty pharmaceutical (e.g., growth hormone) to someone at home. The patient copayment may be much lower for this home-care service, but as a general trend in the last 10 years, health plans have been increasing the copayments for pharmaceuticals as a cost-management technique. ${ }^{7}$ In this scenario, patients may now have a financial disincentive against receiving these specialty pharmaceuticals from the pharmacy benefit.

This dynamic can lead to some challenges for pharmacists within health plans. Some specialty pharmacy providers know exactly what they get paid in the medical and pharmacy benefit. When a pharmacist within a health plan starts bringing the issue of these differences in payment amounts to the staff that manages the medical benefit, it can be a very challenging conversation. As explained previously, the 2 groups are managing different processes, holding different contracts, and paying at different rates. It takes a lot of analysis and mutual learning before a solution comes into focus. The following specific examples of tactics that health plans can use to address this arbitrage (the practice of paying 2 different amounts for the same item) are presented to better understand this phenomenon.

\section{Health Plan Tactics Regarding Specialty Pharmacy}

Despite the constraints of these core process differences between the medical and pharmacy claims payment systems in processing specialty pharmacy payments, health plans are making some significant inroads into managing specialty pharmacy services more effectively.

Tactic 1: Contain a Pharmacy Benefits Management Company. It is relatively common today for health plans to operate a full-service PBM as a free-standing, internal company. This gives the health plan a greater number of pharmacists within the organization to build and manage a network and execute the clinical programs of a full-service PBM. It also gives greater transparency to these arbitrage issues between the claims payment systems. Many of the largest health plans in the country, WellPoint, Aetna, CIGNA, and Humana, have an internal division that serves as their PBM. This gives the organization a critical mass when dealing with issues such as these. This type of organization can support an internal Pharmacy and Therapeutics Committee process and can align itself with the clinical programs and contracting activities on both the medical and pharmacy benefit.
Tactic 2: Build a Specific Specialty Pharmacy Benefit. The current health plan benefit language has been built upon several decades of history but fails to adequately address the issues of specialty pharmacy. Some health plans have created a new benefit type to address specialty pharmacy services that focuses less on site of service and more on the products being distributed. Once the services in the specialty pharmacy benefit are defined, the contracting for these services can be implemented, typically with a narrowing of the network. One of the definitions that has been used in this approach in the past is a "self-injectable pharmaceutical" benefit. By decreasing the number of sources from which patients can obtain these products, health plans are addressing the pricing arbitrage between the many possible procurement pathways.

Tactic 3: Contain a Specialty Pharmacy. Carrying the "build or buy" dilemma to its logical conclusion, several of the larger health plans have taken the step of building specialty pharmacies in house. WellPoint has PrecisionRx Specialty Solutions, Aetna operates Aetna Specialty Management, and Cigna operates Tel-Drug Specialty Pharmacy. A consortium of Blue Cross Blue Shield plans own Prime Therapeutics as a joint venture, and this company in turn owns and manages a specialty pharmacy. The number of lives that the health plans have needed before pursuing this kind of strategy is typically $\geq 9$ million members: WellPoint has approximately 35 million members; Aetna, 12 million; CIGNA, 9 million; and the partners of the Prime Therapeutics joint venture, collectively approximately 16 million.

\section{Conclusions}

Managing the specialty pharmacy benefits successfully is a complex undertaking. Managing specialty pharmacy in the fullest sense of the term requires a familiarity with both the medical and pharmacy benefits, as well as contracts in both benefits. A number of historical factors, such as health plan benefits, provider types, contracts that are specific to provider type, different coding systems, and different pricing files, all contribute to the difference in payments for the same services (i.e., arbitrage) between the medical and pharmacy benefits.

There are several tactics that health plans are currently implementing to manage specialty pharmacy service more effectively, from building a PBM in-house to creating a unique specialty pharmacy benefits to building a specialty pharmacy in-house.

These tactics are designed to bring about greater transparency and scrutiny of specialty pharmacy services and, directly or indirectly, to have these services provided by the most cost-efficient providers. Specialty pharmacy services are being provided by ever-tighter networks, and often a business unit of the health plan and/or a business unit of the health plan's PBM are competitors to the independent specialty pharmacy. The opportunities for contracting with health plans and PBMs may become more complex in the future. Also, with the growth and consolidation of specialty pharmacies, these organizations themselves are now 
large companies that are typically a division within an even larger company.

While the current size and rapid growth-rate of the specialty pharmacy sector suggests that there is tremendous opportunity for pharmacies to enter this space, the tactics undertaken by health plans to manage this area more closely suggest that the opportunity is, in fact, smaller than it appears. For those interested in entering into this space or interested in managing specialty pharmacy services more effectively from the health plan standpoint, there are many operational issues that need to be in alignment. Among those many operational issues, whether or not a pharmacy contracts on the medical benefit is actually a rather small issue. However, failing to consider whether a pharmacy contracts to a specialty pharmacy or to a health plan ignores a substantial amount of the specialty pharmacy opportunity and revenue that make up this dynamic and rapidly growing marketplace.

\section{ACKNOWLEDGMENTS}

The author acknowledges John Aforismo, BScPharm, RPh, President of RJ Health Systems, for his review of earlier versions of this manuscript.

\section{DISCLOSURES}

Robert C. McDonald received compensation from Impact Education, LLC, for the preparation of this article. Also, he had editorial control. McDonald was responsible for the entire study concept and design of this article. He performed all the data collection, data interpretation, writing, and revision of this article.

\section{REFERENCES}

1. AMCP Task Force on Drug Payment Methodologies. AMCP guide to pharmaceutical payment methods. J Manag Care Pharm. 2007;13(8):S1-S40. Available at: www.amcp.org/data/jmcp/JMCPSUPPC_OCT07.pdf.

2. Kunze AM, Gunderson BW, Gleason PP, Heaton AH, Johnson SV. Utilization, cost trends, and member cost-share for self-injectable multiple sclerosis drugs-pharmacy and medical benefit spending from 2004 through 2007. J Manag Care Pharm. 2007;13(9):799-806. Available at: www. amcp.org/data/jmcp/JMCPMaga_N-D\%2007_799-806.pdf.

3. Starr P. The Social Transformation of American Medicine. New York: Basic Books; 1982.

4. Blue Cross and Blue Shield Association. Blues History. Available at: www. bcbs.com/news/press/facts/blues-history.html. Accessed December 30, 2007.

5. Express Scripts. 2006 Drug Trend Report. Available at: www.expressscripts.com/ourcompany/news/industryreports/drugtrendreport/2006/. Accessed December 30, 2007.

6. Centers for Medicare and Medicaid Services. 2005 National Care Expenditures Data. Available at: www.cms.hhs.gov/nationalhealthexpenddata/01_overview.asp. Accessed December 30, 2007.

7. Fendrick AM, Chernew ME. Value-based insurance design: aligning incentives to bridge the divide between quality improvement and cost containment. Am J Manag Care. 2006;12:SP5-SP10. 